\title{
Una Exploración de las Desigualdades Socioeconómicas en Morbilidad en España *
}

\author{
Joan Costa-Font \\ Departament de Teoria Econòmica \& CAEPS, Universitat de Barcelona. \\ LSE Health, London School of Economics, Londres
}

Joan Gil

Departament de Teoria Econòmica \& CAEPS, Universitat de Barcelona.

\begin{abstract}
Resumen
Una de las explicaciones del proceso causal que comporta la persistencia de un vector socio-económico en salud es la existencia previa o simultánea de desigualdades en morbilidad. Este artículo realiza una exploración empírica de la cuestión. En particular se examina la existencia de un patrón de desigualdades socioeconómicas en la incidencia de determinadas enfermedades crónicas responsables de una elevada tasa de mortalidad en España. Para ello hemos aplicado una metodología de descomposición de los índices de concentración sobre una muestra representativa de adultos de la Encuesta Nacional de Salud de 2003. En primer lugar, nuestros resultados revelan la existencia de desigualdad socio-económica (en contra de los situados en la parte baja de la escala de la renta) en la probabilidad de sufrir enfermedades del corazón, hipertensión arterial, diabetes y colesterol elevado. En segundo lugar, la estimación de las funciones de producción confirma que la desigualdad se atribuye a problemas de salud cuya incidencia está ligada a factores demográficos si bien el nivel educativo y la actividad económica (pensionista) son determinantes destacables del grado de desigualdad socioeconómica en morbilidad. Como el nivel de renta individual apenas influye en dichas desigualdades, una mera transferencia de renta no necesariamente supondría una reducción de la desigualdad en salud.
\end{abstract}

Palabras clave: morbilidad, enfermedades crónicas, desigualdades socioeconómicas, tasas de mortalidad, descomposición de la desigualdad.

Clasificación JEL: H51, I19

\begin{abstract}
One of the potential reason underpinning the persistence of a socio-economic vector in health is the previous or simultaneous existence of inequalities in morbidity. This paper empirically examines the existence of a pattern of socio-economic inequalities in the probability of suffering chronic conditions responsible of high mortality rates in Spain. We draw from standard methods of estimation and decomposition of the concentration indexes in its main determinants. We employ the adult sample of the Spanish National Health Survey 2003. First, our results suggest the existence of significant indexes of inequality in the probability of heart diseases, arterial hypertension, diabetes and high

* Los autores quieren agradecer los comentarios y sugerencias de Ángel López-Nicolás a una versión previa de este trabajo. Se reconoce a su vez la ayuda financiera prestada por el Sistema Español de Ciencia y Tecnología (Proyecto No. SEJ2005-06270/ECON) y por la Generalitat de Catalunya (Proyecto No. SGR2005-460) y también al XREPP-Xarxa de Referència en Economia i Polítiques Públiques.
\end{abstract}


cholesterol. However, the health production function confirms that such conditions'incidence and inequality results mainly from the aging process and gender. Finally, besides demographic - gender and age - determinants educational attainment and economic status (pensioner) are also key factors explaining inequalities in morbidity. Hence, as income alone hardly affects these patterns of inequalities a mere income redistribution policy would not necessarily imply an ulterior reduction in health inequality.

Key words: health economics, chronic diseases, socio-economic inequalities, mortality rates.

JEL Classification: H51, I19

\section{Introducción}

La existencia de una asociación entre bajo nivel de renta y mala salud ha sido objeto de algo más de doscientos estudios publicados que forman parte de la literatura de economía de la salud. Especialmente, los estudios empíricos destacan en aquellos países en los que la inequidad y la cohesión social son una prioridad política de primera magnitud ${ }^{1}$. Si bien existe aun amplia heterogeneidad de enfoques metodológicos, parece haber un cierto consenso entre economistas en la utilización del coeficiente de concentración como índice de desigualdad (Wagstaff y Van Doorslaer, 2000) y en la utilización de métodos de regresión para poder descomponer tales índices y examinar así los determinantes de la desigualdad además de la desigualdad en sí misma. En general, todos estos trabajos coinciden en apuntar la existencia de importantes desigualdades socioeconómicas en salud. No obstante, cuando uno se pregunta por las posibles causas puede concluirse que éstas continúan siendo un misterio (o 'black box'). Este trabajo pretender contribuir a esta última cuestión haciendo uso de los métodos desarrollados recientemente en la literatura, pero examinando el papel de la morbilidad y no el resultado final (mala salud).

Entre las diferentes hipótesis de la explicación de la generación de desigualdades en salud destaca un primer grupo de teorías que defiende lo que se denomina 'efecto causal' o «mechanism-driven approach» (Mackenbach, 2005). Este enfoque sostiene que la posición socioeconómica -en la jerarquía de posibilidades de consumo y gasto- de cada individuo es lo que acaba generando desigualdades observadas en salud. De modo que las desigualdades en la exposición a determinados factores de riesgo materiales como la renta - bien sea absoluta (Evans, 2002) o relativa (Wilkinson, 1997) - el tipo de comportamiento (tabaquismo, alcoholismo, nutrición y ejercicio físico inadecuado) o de tipo psico-social (tales como una diferente exposición al stress, pérdida de seres queridos, desequilibrio entre esfuerzo y premio, etc.) más presentes

1 A nivel español destacarían, entre otros, los trabajos de Rodríguez et al. (1993), Regidor et al. (1995), Abasolo (1998), Urbanos (2000, 2001), Gómez-García y López (2004a,b y 2005), Stoyanova (2004), Costa-Font (2005) y Costa-Font y Gil (2008c). En el plano internacional puede consultarse, entre otros, a Wagstaff y Van Doorslaer (2000), Van Doorslaer y Koolman (2004), Van Doorslaer et al. (2004), Kunst et al. (2005) y Mackenbach (2005). 
en los grupos socioeconómicos más bajos, explicaría las desigualdades en la salud (Wilkinson, 1997). Entre las implicaciones de este tipo de desigualdad está la necesidad de reducir la desigualdad socio-económica (por ejemplo, vía mecanismos redistributivos).

Otro grupo de explicaciones pasa por argumentar, siguiendo a Deaton (2002), que las desigualdades en estado de salud son una consecuencia inevitable del hecho que aquellos colectivos relativamente más pobres «producen» comparativamente una menor cantidad de bienes o 'inputs' saludables y, a su vez, están expuestos en mayor medida a hábitos poco saludables (tabaco, alcohol, nutrición). Este tipo de teorías ponen especial énfasis en programas dirigidos a poblaciones con menores recursos («low income targeting») quizás para compensar la falta de información o capacidad de unos colectivos a proteger. Finalmente, otro enfoque en el que se insertaría este trabajo defendería la tesis que las desigualdades en salud son una consecuencia de la desigual distribución de riesgos sanitarios (Link y Phelan, 1995) o de la incidencia de las enfermedades crónicas que, además de ser responsables de muchas muertes prematuras, estarían sujetas a un claro gradiente socioeconómico. Detrás de este argumento está la idea que el vector socio-económico contiene información sobre un diferente acceso a la información sanitaria dependiente de la renta, si bien a menudo no es observable por el investigador, aunque puede observarse un resultado intermedio entre la información y la salud, tal como la enfermedad.

A nivel internacional existe un cierto volumen de literatura que valida la existencia de diferencias económicas en morbilidad ${ }^{2} \mathrm{y}$, recientemente, han aparecido algunos trabajos basados en microdatos que hallan significativas y dispares desigualdades socioeconómicas -medidas por el nivel educativo- en el padecimiento de ciertas dolencias crónicas (declaradas) en países de la $\mathrm{UE}^{3}$, de modo que los individuos de menor nivel educativo soportarían una mayor incidencia de estas enfermedades de «larga duración» que los más educados ${ }^{4}$ (cf. Mackenbach, 2005, y Dalstra et al., 2005). Sin embargo, para el caso de España apenas existen investigaciones de este tipo. Las excepciones a esta pauta son, por un lado, los trabajos sobre salud mental medida a través del test GHQ para la población catalana (GarcíaGómez y López, 2005) y sobre depresión declarada para la población española (Costa-Font y Gil, 2008b). Ambos trabajos demuestran que las decilas de renta inferiores disfrutan de un peor estado de salud mental (una mayor incidencia del trastorno depresivo), siendo los ingresos familiares el principal predictor del grado de desigualdad junto a la actividad laboral y el nivel educativo. Por otro lado, el estudio de Dalstra et al. (2005) que documentan la existencia en España de destacables desigualdades en educación con unos valores -estadísticamente significativos- de la OR igual a 1,99 para el caso de la diabetes 'mellitus' y 1,82 para las enfermeda-

\footnotetext{
Al respecto pueden consultarse las referencias citadas en Dalstra et al. (2005).

Sería, principalmente, el caso de los accidentes cerebrovasculares ('stroke'), enfermedades del sistema nervioso, diabetes y artritis con unos 'odd ratios' (OR) superiores a 1,50.

4 Siendo esto válido tanto entre la población en edad de trabajar como en los jubilados y entre hombres y mujeres.
} 
des crónicas respiratorias. Sin embargo, las OR calculadas para los accidentes cerobrovasculares, la hipertensión arterial o las enfermedades del corazón no son significativamente distintos de la unidad. ${ }^{5}$

Este artículo se propone medir y explicar las diferencias en el grado de desigualdad relacionado con la renta en la probabilidad de sufrir determinadas enfermedades crónicas declaradas responsables de una alta tasa de mortalidad en España (i.e., enfermedades del corazón, hipertensión arterial, diabetes y colesterol elevado) por medio de aplicar - a diferencia de Dalstra et al., 2005- métodos de regresión recientemente elaborados (Van Doorslaer y Koolman, 2002) y el empleo de la Encuesta Nacional de Salud de 2003. En otros términos, las cuestiones a examinar en este artículo son tres: averiguar si existe un patrón de desigualdades, analizar su grado de homogeneidad y, finalmente, ver hasta qué punto obedecen a causas parecidas. Para ello la Sección 2 está dedicada a cuantificar la dimensión del problema estudiado, la Sección 3 presenta los datos, la medición de enfermedades crónicas empleada y el método seguido para cuantificar y descomponer el indicador de desigualdades propuesto, la Sección 4 presenta los principales resultados y, finalmente, la Sección 5 concluye a modo de discusión.

\section{Morbilidad y enfermedades crónicas: evidencia y patrón socio-económico}

Existe una cierta imprecisión terminológica, de la cual no nos escaparemos, acerca del significado concreto de «enfermedades crónicas» y su importancia como proxy de morbilidad. Así, mientras que algunas condiciones consideradas como crónicas pueden catalogarse como agudas (ciertas enfermedades del corazón), otras clasificadas como comunicables (como el VIH/SIDA) son también enfermedades con «características crónicas». En este trabajo siguiendo a la OMS (2005) nos referiremos, sin embargo, al término 'enfermedades crónicas' en lugar del también empleado 'enfermedades no-comunicables' al dar cuenta de una enfermedad de larga duración cuyo tratamiento también requiere seguir un enfoque a largo plazo ${ }^{6}$.

Tradicionalmente se ha venido creyendo que las enfermedades crónicas eran 'enfermedades de la opulencia’ afectando básicamente a los países ricos. Sin embargo, los últimos datos epidemiológicos disponibles vienen a invalidar esta visión, a la par que contribuyen a corregir tanto la cuantificación de los costes reales impuestos por las enfermedades crónicas como la ponderación política sobre su prevención y control (OMS, 2005 y Suhrcke et al., 2006). Así, según Mathers et al. (2003) mientras

5 Respecto a las desigualdades socioeconómicas en la exposición a factores de riesgo (como la obesidad) que pueden dar lugar a determinadas enfermedades crónicas puede consultarse a Costa-Font y Gil (2008a).

6 Vale la pena reseñar que si bien las enfermedades no-comunicables no son transmisibles biológicamente, los factores de riesgo pueden perfectamente transmitirse. Es lo que Surhcke et al. (2005) denominan 'peers effects' o la influencia de los comportamientos de personas cercanas al individuo en cuestión (i.e., abuso de sustancias en los adolescentes o ejercicio físico y dieta). 
que las muertes debidas a la incidencia de las enfermedades crónicas en el año 2002 en los países de renta alta eran cercanas al 90\%, en los países de renta media eran del $73-75 \%$ y en los países de renta baja un considerable $41 \%{ }^{7}$. Además, según prevé la OMS hacia el año 2015 las enfermedades de larga duración ya serán las principales responsables de las muertes en los países de renta baja (Suhrcke et al., 2006). Otra idea que los datos desmienten se refiere a que las enfermedades crónicas no son un problema exclusivo de las personas mayores sino que también afecta a la población en edad de trabajar, sobre todo en los países de renta media y baja. De acuerdo con Mathers et al. (2003) hasta un 44\% (33\%) de las muertes por enfermedades crónicas en los países de renta baja (media) tienen lugar en edades anteriores a los 60 años. Ese porcentaje es de cerca del $20 \%$ en los países ricos. Por último, en los países de renta alta apenas existen dudas que las enfermedades crónicas inciden primordialmente sobre las espaldas de los pobres, siendo la evidencia mucho menos clara en los países pobres en gran parte debido a la falta de datos de encuestas fiables (Mackenbach, $2005)^{8}$.

Según la OMS (2005) un 52\% (35 millones) del total de defunciones por todas las causas previstas en el 2005 en todo el mundo fueron producidas por cuatro enfermedades crónicas: enfermedades cardiovasculares (principalmente enfermedades del corazón y accidentes cerebrovasculares), cáncer, enfermedades pulmonares crónicas y diabetes ${ }^{9}$. Existe una amplia evidencia que muestra que los principales factores de riesgo de padecer estas dolencias crónicas son, básicamente, el tabaco, una pobre dieta, la falta de ejercicio físico y la obesidad. Dada esta relevancia, este trabajo se centrará concretamente en estudiar las desigualdades socioeconómicas en la prevalencia de cuatro problemas de salud crónicos -enfermedades del corazón, hipertensión arterial, diabetes y colesterol elevado- que por otro lado, tienen la ventaja de presentar entre una moderada a alta coincidencia entre los datos declarados por los entrevistados en las encuestas y los basados en historiales médicos (cf., ente otros trabajos, Tormo et al., 2000, y Goldman et al., 2003). Según la Tabla 1 las enfermedades del corazón (infarto de miocardio, angina de pecho, etc.) afectan a un $6,2 \%$ de la población adulta española, la diabetes a un $6,3 \%$, el colesterol elevado a un $10,8 \%$ y la hipertensión arterial a un $15,6 \%$. Por genero, la incidencia de dichas enfermedades es ligeramente mayor para las mujeres, con la excepción de la hipertensión arterial que es bastante mayor (18\% vs. 13\%) y por edades la incidencia es casi testimonial para la población menor a 45 años y muy significativa para los mayores de 65 años.

Este último dato es engañoso ya que el factor que sitúa el peso de las enfermedades crónicas por debajo del $50 \%$ en los países pobres es la elevadísima cifra sobre enfermedades comunicables o infecciosas, maternales y prenatales y deficiencias nutritivas registradas en la región del África sub-sahariana.

8 Aunque existe suficiente evidencia respecto a algunos factores de riesgo que muestra, sin ambigüedad, que la prevalencia del tabaco o la obesidad en países de renta baja es mayor entre los pobres.

9 Un $9 \%$ se deben al resto de problemas crónicos, un 30\% a las enfermedades infecciosas y el resto a accidentes. 
TABLA 1

TASA DE PREVALENCIA DE DETERMINADAS ENFERMEDADES CRÓNICAS EN LA POBLACIÓN ADULTA EN 2003

\begin{tabular}{|l|r|r|r|r|}
\hline & $\begin{array}{c}\text { Enfermedades } \\
\text { Corazón }\end{array}$ & $\begin{array}{c}\text { Hipertensión } \\
\text { Arterial }\end{array}$ & Diabetes & $\begin{array}{c}\text { Colesterol } \\
\text { Elevado }\end{array}$ \\
\hline Total & $6,19 \%$ & $15,56 \%$ & $6,32 \%$ & $10,79 \%$ \\
Hombres & $5,96 \%$ & $13,28 \%$ & $5,85 \%$ & $9,95 \%$ \\
Mujeres & $6,41 \%$ & $17,70 \%$ & $6,77 \%$ & $11,59 \%$ \\
Edad 16-29 & $0,70 \%$ & $0,83 \%$ & $0,50 \%$ & $1,26 \%$ \\
Edad 30-44 & $0,85 \%$ & $3,99 \%$ & $1,00 \%$ & $4,50 \%$ \\
Edad 45-64 & $6,28 \%$ & $20,79 \%$ & $7,91 \%$ & $16,89 \%$ \\
Edad 65+ & $19,90 \%$ & $42,10 \%$ & $18,47 \%$ & $22,95 \%$ \\
\hline
\end{tabular}

Nota: Los cálculos emplean los factores de elevación de la encuesta.

Fuente: Encuesta Nacional de Salud de 2003 (INE).

\section{Datos y Método}

\subsection{Los datos}

Los datos empleados en este trabajo se obtienen de la Encuesta Nacional de Salud (ENS) de 2003 elaborada bienalmente por el INE, en colaboración con el Ministerio de Sanidad y Consumo, con representatividad nacional y por comunidades autónomas y que va dirigida a las familias con la finalidad de proporcionar información sobre el estado de salud, enfermedades crónicas, accidentalidad, hábitos de vida, utilización de servicios sanitarios, prácticas preventivas, etc., a partir de las respuestas ofrecidas por los encuestados. La ENS se realizada en dos fases. En la primera se capta a todos los individuos del hogar solicitándoles información socio-económica y demográfica (Cuestionario del Hogar); en la segunda se recoge información de un adulto (16 y más años) seleccionado aleatoriamente (Cuestionario de Adultos) y, si hubiere, se selecciona también del mismo modo un menor de edad (Cuestionario de Menores).

La técnica de muestreo utilizada es de tipo 'polietápica' estratificada. Las unidades de primera etapa son las secciones censales (del Padrón Municipal de habitantes de 2002) que se agrupan en estratos de acuerdo con el tamaño del municipio al que pertenece la sección; y las unidades de la segunda etapa son las viviendas familiares principales, investigándose a todos los hogares que tienen su residencia habitual en las mismas. Para poder facilitar estimaciones con un determinado grado de fiabilidad a nivel nacional y de CCAA, se ha seleccionado una muestra de aproximadamente 22.000 viviendas distribuidas en 1.844 secciones censales. La muestra se distribuye entre CCAA asignando una parte uniformemente y otra proporcionalmente al tamaño de cada CCAA. 
La muestra original contiene 21.650 adultos ( $48,8 \%$ hombres, $51,2 \%$ mujeres) con edades comprendidas entre los 16-99 años. Desafortunadamente, se eliminaron 5.483 observaciones al no figurar ningún valor en la variable de ingresos mensuales, con lo que finalmente nuestra muestra constará de 16.167 observaciones después de examinar los posibles efectos de selección muestral. ${ }^{10}$

La variable ingresos mensuales (o variable ranking) es nuestra 'proxy' del status socioeconómico (SES) del hogar. ${ }^{11}$ En concreto, la renta total neta del hogar se recoge a través de una variable intervalo con hasta 8 categorías o respuestas. Se decidió aplicar un modelo de regresión intervalo utilizando información socioeconómica del cabeza de familia (edad, género, nivel de estudios, ocupación) y del propio hogar (número de adultos o seguros médicos privados) para derivar una medida continua de la renta familiar. Tras asignar una renta neta a cada hogar, ésta se transformó dividiendo por un factor de equivalencia (el número de miembros del hogar elevado a $1 / 2$ ) para tener en cuenta las diferencias de tamaño y composición de los hogares.

\subsection{Estimación de la morbilidad declarada: enfermedades crónicas}

Nuestra medida de cada una de las enfermedades crónicas (por lo tanto no debidas a procesos agudos aislados) que se estudian en este trabajo deriva de las respuestas a una lista de hasta 16 enfermedades o problemas de salud crónicos diagnosticados por el médico y que la ENS plantea a cada individuo entrevistado. En concreto la pregunta se formula en los siguientes términos: «¿En los últimos doce meses, le ha dicho su médico que padece de forma crónica alguna de las siguientes enfermedades o problemas de salud en la actualidad?». Las respuestas binarias ofrecidas a cada una de esas cuatro enfermedades constituyen nuestra medida de enfermedad crónica declarada.

A continuación se transformó cada medida de enfermedad crónica declarada $\left(y_{i}\right)$ en una variable continua tras utilizar las predicciones de un modelo lineal de probabilidad (MLP) estimado mediante MCO del tipo,

$$
y_{i}=\alpha+\sum_{k} \beta_{k} x_{k, i}+\varepsilon_{i}
$$

donde $y_{i}=1$ (si el individuo declara que padece una enfermedad crónica), $\varepsilon_{i}$ es el término de error, $x_{k}$ es un conjunto de determinantes exógenos de las enfermeda-

10 La omisión de esta información tiende, sin embargo, a elevar aunque muy ligeramente la tasa de prevalencia de nuestras variables dependientes: enfermedades del corazón (5,84\% muestra entera, $6.19 \%$ muestra restringida), hipertensión arterial ( $14,53 \%$ vs. $15,56 \%)$, diabetes $(5,92 \%$ vs. $6,32 \%)$ y colesterol elevado (10,46\% vs. $10,79 \%)$.

11 Otras variables pueden igualmente aproximar la posición socioeconómica de cada familia, es el caso de la educación o el tipo de empleo. Sin embargo, el método de descomposición seguido exige una medida continua del SES. 
des crónicas y $\beta_{k}$ el conjunto de parámetros asociados. De esta especificación se deriva la siguiente medida de la probabilidad de padecer una determinada condición crónica,

$$
P\left(y_{i}=1\right)=\alpha+\sum_{k} \beta_{k} x_{k, i}
$$

La opción por ajustar un MLP (que proporciona estimaciones consistentes de los parámetros $\beta_{k}$ ) se explica por el hecho que la linealidad en parámetros es una propiedad deseada para poder, posteriormente, descomponer el índice de desigualdad de la probabilidad de sufrir una determinada enfermedad crónica (Cf. Van Doorslaer y Koolman, 2004, Van Doorslaer et al., 2004, García-Gómez y López 2004 a,b).

Siguiendo las pautas de la literatura, cada modelo de regresión estimado para las cuatro enfermedades crónicas analizadas se ha ajustado al siguiente conjunto de variables explicativas (ver Tabla 2): a) el logaritmo de la renta neta familiar equivalente; b) ocho variables 'dummy' de edad y género correspondientes a los grupos 16$29,30-44,45-64,65$ y más años para hombres y mujeres. Estas variables demográficas constituyen, para nuestros propósitos, lo que puede considerarse como determinantes de la desigualdad inevitable; c) cuatro categorías de estado civil que recogerán, genéricamente, los efectos de las relaciones familiares sobre cada tipo de enfermedad; d) tres variables 'dummy' de nivel educativo para medir el impacto del conocimiento individual sobre los riesgos de la salud; e) ocho categorías sobre la condición o el estatus laboral; f) un conjunto de variables dicotómicas sobre estilos de vida con claras conexiones sobre el estado de salud de la población, en concreto tres 'dummy' sobre el hábito de fumar, una sobre el habito de beber alcohol, cuatro sobre la frecuencia de realizar actividad física en el lugar de trabajo, hogar, etc., y, finalmente, otra 'dummy' sobre la realización de ejercicio físico en tiempo libre. Por último, dado que España es un país heterogéneo se hace, ciertamente, necesario controlar por las diferencias que existan tanto a nivel cultural como en términos alimentarios: por tanto nuestra estimación incorpora g) dieciocho variables regionales o 'dummy' para cada CCAA ${ }^{12}$.

El modelo econométrico subrayado no debiera contemplarse como un modelo estructural de enfermedades crónicas, ni tampoco debiera interpretarse los resultados de modo causal. Alternativamente, debiera verse como una ecuación de regresión en forma reducida que evidencia cómo cambios en los determinantes de cada enfermedad crónica termina afectando el grado de desigualdad socio-económica observado en cada una de ellas. La Tabla 2 presenta las definiciones de las variables empleadas, la media y el error estándar como estadísticos descriptivos.

12 Este conjunto de regresores se han mostrado, por otro lado, adecuados como predictores de otras enfermedades crónicas por parte de los autores (Costa-Font y Gil, 2008a y 2008b). 
TABLA 2

DESCRIPCIÓN DE VARIABLES, VALORES MEDIOS Y ERRORES ESTÁNDAR

\begin{tabular}{|c|c|c|c|}
\hline Variables & Definición & Media & $\begin{array}{c}\text { E.S. } \\
\text { (media) }\end{array}$ \\
\hline \multicolumn{4}{|l|}{ Dependientes: } \\
\hline Enfermed. del corazón & Var. 'dummy': 1 Tiene diagnostic. enfermedad del corazón; 0 otro & 0,0619 & 0,0019 \\
\hline Hipertensión arterial & VVr. 'dummy': 1 Tiene diagnosticado hipertensión arterial; 0 otro & 0,1556 & 0,0028 \\
\hline Diabetes & Var. 'dummy': 1 Tiene diagnosticado diabetes; 0 otro & 0,0632 & 0,0019 \\
\hline Colesterol elevado & Var. 'dummy': 1 Tiene diagnosticado colesterol elevado; 0 otro & 0,1079 & 0,0024 \\
\hline \multicolumn{4}{|l|}{ Independientes: } \\
\hline Log. Renta Equival. & Logaritmo de la renta familiar neta equivalente & 6,5155 & 0,0044 \\
\hline M16-29 & Var. 'dummy': 1 Varones de $16-29$ años; 0 otro & 0,1167 & 0,0025 \\
\hline M30-44 & Var. 'dummy': 1 Varones de $30-44$ años; 0 otro & 0,1516 & 0,0028 \\
\hline M45-64 & Var. 'dummy': 1 Varones de $45-64$ años: 0 otro & 0,1290 & 0,0026 \\
\hline M65+ & Var. 'dummy': 1 Varones de 65 y más años; 0 otro & 0,0879 & 0,0022 \\
\hline F30-44 & Var. 'dummy': 1 Mujeres de 30-44 años; 0 otro & 0,1452 & 0,0028 \\
\hline F45-64 & Var. 'dummy': 1 Mujeres de $45-64$ años; 0 otro & 0,1301 & 0,0026 \\
\hline $\mathrm{F} 65+$ & Var. ‘dummy': 1 Mujeres de 65 y más años; 0 otro & 0,1207 & 0,0026 \\
\hline Casado & Var. 'dummy': 1 Casados; 0 otro & 0,5782 & 0,0039 \\
\hline Viudo & Var. 'dummy': 1 Viudos; 0 otro & 0,0748 & 0,0021 \\
\hline Divorciado & Var. 'dummy': 1 Divorciados y sep. legalmente; 0 otro & 0,0269 & 0,0013 \\
\hline Educación baja & Var. 'dummy': 1 Educación primaria o inferior; 0 otro & 0,4493 & 0,0039 \\
\hline Educación media & Var. 'dummy': 1 Educación media/secundaria; 0 otro & 0,4065 & 0,0039 \\
\hline Autónomo & Var. 'dummy': 1 Trabajador por cuenta propia; 0 otro & 0,0726 & 0,0020 \\
\hline Empleado público & Var. ‘dummy': 1 Empleado público; 0 otro & 0,0838 & 0,0022 \\
\hline Serv. doméstico & Var. ‘dummy': 1 Empleado en servicio doméstico; 0 otro & 0,4502 & 0,0039 \\
\hline Pensionista & Var. ‘dummy': 1 Pensionista no invalidez; 0 otro & 0,1857 & 0,0031 \\
\hline Pensionista invalidez & Var. 'dummy': 1 Pensionista de invalidez; 0 otro & 0,0405 & 0,0016 \\
\hline 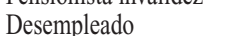 & Var. 'dummy': 1 Desempleado; 0 otro & 0,0833 & 0,0022 \\
\hline Inactivo voluntario & Var. 'dummy': 1 Inactivo voluntario; 0 otro a & 0,2877 & 0,0036 \\
\hline Ex-fumador & Var. 'dummy': 1 Fumador en el pasado; 0 otro & 0,1713 & 0,0030 \\
\hline No fumador & Var. 'dummy': 1 Nunca ha fumado; 0 otro & 0,5265 & 0,0039 \\
\hline Bebedor & Var. ‘dummy': 1 Ha consumido bebidas alcohólicas... últimos 12 meses.; 0 otro & 0,5616 & 0,0039 \\
\hline Baja actividad física & Var. "dummy': 1 Baja activ. física en el trabajo, hogar, etc.; 0 otro ${ }^{b}$ & 0,4798 & 0,0039 \\
\hline Moderada actividad & Var. 'dummy': 1 Moderada activ. física en el trabajo, hogar, etc.; física 0 otro ${ }^{\circ}$ & 0,1216 & 0,0026 \\
\hline Alta actividad física & Var. 'dummy': 1 Elevada activ. física en el trabajo, hogar, etc.; 0 otro ${ }^{\mathrm{C}}$ & 0,0559 & 0,0018 \\
\hline Act. física ocio & Var. 'dummy': 1 Realiza alguna actividad física 0 deportiva en tiempo libre: 0 otro & 0,4048 & 0,0039 \\
\hline Aragón & Var. 'dummy': 1 Residente en Aragón; 0 otro & 0,0177 & 0,0010 \\
\hline Asturias & Var. "dummy': 1 Residente en Asturias; 0 otro & 0,0332 & 0,0014 \\
\hline Baleares & Var. 'dummy': 1 Residente en Islas Baleares; 0 otro & 0,0256 & 0,0012 \\
\hline Canarias & Var. “dummy': 1 Residente en Islas Canarias; 0 otro & 0,0584 & 0,0018 \\
\hline Cantabria & Var. 'dummy': 1 Residente en Cantabria; 0 otro & 0,0161 & 0,0010 \\
\hline Castilla-León & Var. ‘dummy': 1 Residente en Castilla y León; 0 otro & 0,0588 & 0,0018 \\
\hline Castilla-Mancha & Var. 'dummy': 1 Residente en Castilla La Mancha; 0 otro & 0,0503 & 0,0017 \\
\hline Cataluña & Var. ‘dummy': 1 Residente en Cataluña; 0 otro & 0,1074 & 0,0024 \\
\hline C. Valenciana & Var. 'dummy': 1 Residente en Comunidad Valenciana; 0 otro & 0,1029 & 0,0024 \\
\hline Extremadura & Var. 'dummy': 1 Residente en Extremadura: 0 otro & 0,0279 & 0,0013 \\
\hline Galicia & Var. 'dummy': 1 Residente en Galicia; 0 otro & 0,0841 & 0,0022 \\
\hline Madrid & Var. 'dummy': 1 Residente en Madrid; 0 otro & 0,0915 & 0,0023 \\
\hline Murcia & Var. ‘dummy': 1 Residente en Murcia; 0 otro & 0,0277 & 0,0013 \\
\hline Navarra & Var. 'dummy': 1 Residente en Navarra; 0 otro & 0,0163 & 0,0010 \\
\hline País Vasco & Var. 'dummy': 1 Residente en el País Vasco; 0 otro & 0,0626 & 0,0019 \\
\hline Ceuta & Var. 'dummy': 1 Residente en Ceuta; 0 otro & 0,0021 & 0,0003 \\
\hline Melilla & Var. 'dummy': 1 Residente en Melilla; 0 otro & 0,0031 & 0,0004 \\
\hline
\end{tabular}

Nota: a) Recoge los colectivos estudiantes, personas dedicadas a labores del hogar, al voluntariado y las que no ejercen actividad económica alguna; b) De pie la mayor parte de la jornada sin efectuar grandes desplazamientos o esfuerzos; c) Caminando, llevando algún peso, efectuando desplazamientos frecuentes que no requieran gran esfuerzo fisco; d) Realizando tareas que requieren gran esfuerzo físico. Los estadísticos media y error estándar de la media se han calculado utilizando los pesos 'muestrales' de la ENS. Las categorías omitidas son: mujeres de 16-29 años, soltero, educación superior, empleado sector privado, fumador, sedentario, residente en Andalucía. 


\subsection{Medida de la desigualdad}

En este trabajo se emplea el índice de concentración (IC) de cada enfermedad crónica como nuestra medida de la desigualdad relacionada con la renta con respecto a cada problema crónico (Van Doorslaer y Koolman, 2004). El IC de la probabilidad de padecer una enfermedad crónica respecto la renta, concepto similar al índice de Gini ${ }^{13}$, se puede calcular a partir de microdatos siguiendo el método de la covarianza (Jenkins, 1988) como,

$$
I C=\left(\frac{2}{\bar{y}}\right) \operatorname{cov}\left(y_{i}, R_{i}\right)
$$

donde $\bar{y}$ es la tasa de prevalencia media de cada enfermedad crónica, $R_{i}$, es el rango fraccional de la renta del individuo i-ésimo (o la proporción acumulada de la población ordenada por nivel renta hasta el individuo i-ésimo) y $\operatorname{cov}(\cdot)$ expresa el operador covarianza. El IC varía en un intervalo de valores que van desde un mínimo de -1 hasta un máximo de +1 . Estos valores extremos significan que cada una de las enfermedades crónicas estudiadas estaría totalmente concentrada en la persona más pobre y más rica de la población, respectivamente. Por otro lado, un IC igual a cero significa que cada individuo de la población padece la misma tasa de prevalencia de cada enfermedad crónica o, en otros términos, cada tipo de dolencia crónica está distribuida de igual modo para todo nivel de renta.

Siguiendo el trabajo de Wagstaff et al. (2003) existe un modo directo para descomponer el grado de desigualdad socioeconómica en un cada uno de los determinantes o predictores de las enfermedades crónicas. Ello requiere el ajuste de un MLP a cada enfermedad crónica frente a un conjunto de regresores $x_{k}$ como se describía en la ecuación (1). En ese caso, el IC de la probabilidad de sufrir una dolencia crónica puede expresarse como,

$$
I C=\sum_{k}\left(\beta_{k} \frac{\bar{x}_{k}}{\bar{y}}\right) C_{k}
$$

donde $\bar{x}_{k}$ es el valor medio de $x_{k}$ y $C_{k}$ es el índice de concentración de $x_{k}$ frente a la renta. El término entre paréntesis de la ecuación (4) expresa, entonces, la elasticidad de la probabilidad de $y$ (enfermedad crónica) con respecto al regresor $x_{k}$ (evaluado en la media poblacional). Por consiguiente, si se define la estimación de la elasticidad de la probabilidad de una determinada condición crónica con respecto al determinante $k$ como,

$$
\hat{\mu}_{k} \equiv \frac{\hat{\beta}_{k} \bar{x}_{k}}{\bar{y}}
$$

13 Estas medidas de desigualdad difieren en el hecho que la variable ranking es la renta en caso del IC; en cambio para el caso del índice de Gini esa variable es una enfermedad crónicas determinada. 
podemos interpretar el IC como una suma ponderada de la desigualdad estimada en cada uno de sus determinantes, siendo los «pesos» la elasticidad calculada de cada enfermedad crónica respecto a su particular determinante,

$$
\hat{I} C=\sum_{k} \hat{\eta}_{k} \hat{C}_{k}
$$

Esta descomposición, que sigue a Van Doorslaer y Koolman (2004), presenta la ventaja de mostrar claramente la contribución de cada determinante de una determinada enfermedad crónica a la desigualdad total de la misma en dos partes: a) por su impacto sobre cada condición crónica medido a través de $\eta_{k} \mathrm{y}$ b) por su grado de desigualdad respecto la renta, $\left(C_{k}\right)$. Por último, señalar que de acuerdo con Kakwani et al. (1997) la desigualdad total con respecto a una enfermedad crónica específica puede, a su vez, dividirse en dos componentes: desigualdad «potencialmente evitable» y «desigualdad inevitable o intrínseca». El componente inevitable de la desigualdad puede atribuirse, por ejemplo, a las diferencias debidas a la edad y al sexo respecto a la renta, esto es, a la especificidad demográfica de la colectividad. Siguiendo a estos mismos autores, por medio de un método de estandarización indirecto es posible estimar la desigualdad potencialmente evitable (I*) calculando, primero, la desigualdad esperada por motivos puramente demográficos (IC*) y sustrayendo esta influencia al IC global ( $\left.\mathrm{I}^{*}=\mathrm{IC}-\mathrm{IC}^{*}\right)$. De este modo, si $y$ se explica exclusivamente mediante los determinantes edad y sexo de la población, entonces $\hat{I C}$ de la ecuación (6) es idéntico a $C^{*}$, con lo cual la desigualdad potencialmente evitable estimada es nula $\mathrm{o}$, en otras palabras, toda la desigualdad prevista es del tipo inevitable o intrínseca.

\section{Resultados}

\subsection{Análisis de regresión}

La Tabla 3 presenta los resultados de la estimación del MLP para cada una de las cuatro enfermedades crónicas estudiadas por medio de MCO. El valor del estadístico de ajuste $\mathrm{R}^{2}$ es relativamente alto para las cuatro ecuaciones estimadas, tratándose de datos de corte transversal, y el test $\mathrm{F}$ nos confirma la significación conjunta de todos los coeficientes de las variables independientes ${ }^{14}$. Lo más significativo de los resultados expuestos es la existencia de un similar patrón de incidencia por lo que respecta a los determinantes de las distintas enfermedades crónicas estudiadas. Así,

14 Las categorías de referencia son: mujeres de 16-29 años, soltero, educación superior, empleado sector privado, fumador, sedentario y residente en Andalucía. Los resultados indican que la probabilidad de que este individuo de referencia declare padecer enfermedades del corazón es de 0.095, diabetes 0.109 , siendo nula para los otros dos problemas crónicos. 
TABLA 3

ESTIMACIÓN DE LA TASA DE PREVALENCIA E ÍNDICES DE CONCENTRACIÓN $\left(C_{k}\right)$ DE LOS DETERMINANTES DE LAS ENFERMEDADES CRÓNICAS

\begin{tabular}{|c|c|c|c|c|c|}
\hline & Mo & 0 Lineal de Pro & ilidad (Coefí & & \\
\hline & $\begin{array}{l}\text { Enfermedades } \\
\text { corazón }\end{array}$ & $\begin{array}{l}\text { Hipertensión } \\
\text { arterial }\end{array}$ & Diabetes & $\begin{array}{c}\text { Colesterol } \\
\text { elevado }\end{array}$ & $\mathrm{C}_{\mathrm{k}}$ \\
\hline Log. Renta Eq. & $-0,00920^{*}$ & $-0,01039$ & $-0,01221^{* *}$ & $-0,01243^{*}$ & $0,04579^{* *}$ \\
\hline M16-29 & $0,00778^{* *}$ & 0,00280 & 0,00578 & $-0,00679$ & $0,06938^{* *}$ \\
\hline M30-44 & $-0,00027$ & $0,02890^{* *}$ & 0,00706 & $0,02014^{* *}$ & $0,11722^{* *}$ \\
\hline M45-64 & $0,03434^{* *}$ & $0,14504^{* *}$ & $0,05325^{* *}$ & $0,10346^{* *}$ & $0,10677^{* *}$ \\
\hline M65+ & $0,10381^{* *}$ & $0,21478^{* *}$ & $0,12835^{* *}$ & $0,07840^{* *}$ & $-0,24264^{* *}$ \\
\hline F30-44 & $-0,01061^{* *}$ & 0,00177 & $-0,01007^{* *}$ & $-0,00880$ & $0,11544^{* *}$ \\
\hline F45-64 & $0,02596^{* *}$ & $0,15213^{* *}$ & $0,04752^{* *}$ & $0,10934^{* *}$ & $0,04814^{* *}$ \\
\hline F65+ & $0,10872^{* *}$ & $0,34389^{* *}$ & $0,13444^{* *}$ & $0,18437^{* *}$ & $-0,37886^{* *}$ \\
\hline Casado & $0,01509^{* *}$ & $0,03561^{* *}$ & $0,01788^{* *}$ & $0,03262^{* *}$ & $0,01422^{* *}$ \\
\hline Viudo & $0,06074^{* *}$ & $0,05048^{* *}$ & 0,02333 & $-0,02023$ & $-0,37892^{* *}$ \\
\hline Divorciado & $0,04216^{* *}$ & 0,01073 & $0,02980^{* *}$ & 0,03082 & $-0,03633$ \\
\hline Educación baja & 0,00425 & $0,04852^{*}$ & $0,03858^{* *}$ & $0,03741^{*}$ & $-0,25519^{* *}$ \\
\hline Educación media & $-0,01012^{* *}$ & 0,01242 & 0,00526 & 0,00269 & $0,10879^{* *}$ \\
\hline Autónomo & $-0,00559$ & $-0,00426$ & 0,00221 & 0,00125 & $0,18100^{* *}$ \\
\hline Empleado público & $-0,00797^{*}$ & $-0,01145$ & 0,00254 & 0,00542 & $0,48359^{* *}$ \\
\hline Serv. doméstico & $-0,01338^{*}$ & $-0,00705$ & $-0,00844$ & 0,01413 & $0,20453^{* *}$ \\
\hline Pensionista & $0,03781^{* *}$ & $0,05739^{* *}$ & 0,00002 & $0,05422^{* *}$ & $-0,27429^{* *}$ \\
\hline Pension. invalidez & $0,07381^{* *}$ & $0,09899^{* *}$ & $0,04701^{* *}$ & $0,09527^{* *}$ & $-0,24853^{* *}$ \\
\hline Desempleado & $-0,00362$ & 0,00430 & $-0,00178$ & 0,01049 & $-0,19200^{* *}$ \\
\hline Inactivo & $-0,01090$ & 0,00263 & $-0,00042$ & 0,01495 & $-0,10931^{* *}$ \\
\hline Ex-fumador & $0,04285^{* *}$ & $0,03856^{* *}$ & 0,00438 & 0,01555 & $0,06354^{* *}$ \\
\hline No fumador & $0,00813^{* *}$ & $0,02957^{* *}$ & 0,00064 & 0,00223 & $-0,05248^{* *}$ \\
\hline Bebedor & $-0,02068^{* *}$ & $-0,00774$ & $-0,02020^{* *}$ & 0,00319 & $0,08764^{* *}$ \\
\hline Baja activ. física & $-0,02685^{* *}$ & $-0,01676^{* *}$ & $-0,02474^{* *}$ & $-0,01093$ & $-0,05024^{* *}$ \\
\hline Mod. activ. física & $-0,02417^{* *}$ & $-0,03150^{* *}$ & $-0,02600^{* *}$ & $-0,01147$ & $-0,00225$ \\
\hline Alta activ. física & $-0,02714^{* *}$ & $-0,04584^{* *}$ & $-0,03747^{* *}$ & $-0,01151$ & 0,01870 \\
\hline Act. física ocio & $-0,00756^{*}$ & 0,00832 & $-0,00051$ & $0,01524^{*}$ & $0,08272^{* *}$ \\
\hline Aragón & 0,00476 & 0,00665 & $-0,03596^{* *}$ & $-0,00567$ & 0,00156 \\
\hline Asturias & $0,02397^{* *}$ & $-0,01050$ & $-0,04508^{* *}$ & $-0,01452$ & $0,14156^{* *}$ \\
\hline Baleares & $-0,00217$ & 0,01047 & 0,00852 & $-0,01248$ & $0,34823^{* *}$ \\
\hline Canarias & 0,00595 & $0,05218^{* *}$ & 0,00359 & $0,04538^{* *}$ & $-0,25528^{* *}$ \\
\hline Cantabria & $-0,02609^{* *}$ & $-0,00375$ & $-0,03480^{* *}$ & $-0,03947^{* *}$ & $0,16020^{* *}$ \\
\hline Castilla-León & $-0,00662$ & $-0,00737$ & $-0,03575^{* *}$ & $-0,00129$ & $-0,21718^{* *}$ \\
\hline Castilla-Mancha & $0,02187^{* *}$ & $0,04202^{* *}$ & $-0,00189$ & $0,02465^{*}$ & $-0,16600^{* *}$ \\
\hline Cataluña & $0,01539^{*}$ & $0,02762^{* *}$ & $-0,01609$ & 0,01499 & $0,13080^{* *}$ \\
\hline C. Valenciana & 0,00387 & $0,05436^{* *}$ & $-0,00092^{*}$ & $0,03188^{* *}$ & $-0,02164^{* *}$ \\
\hline Extremadura & 0,01467 & $0,05878^{* *}$ & $-0,00906$ & 0,01739 & $-0,37129^{* *}$ \\
\hline Galicia & $0,02521^{* *}$ & $0,04440^{* *}$ & $-0,00987$ & $0,06888^{* *}$ & 0,00682 \\
\hline Madrid & $-0,00171$ & 0,00925 & $-0,01523$ & 0,00866 & $0,18569^{* *}$ \\
\hline Murcia & $-0,00075$ & $0,05358^{* *}$ & $-0,01854^{*}$ & $-0,00229$ & $-0,27464^{* *}$ \\
\hline Navarra & $0,02030^{*}$ & 0,01659 & $-0,02472^{* *}$ & 0,00632 & $0,51618^{* *}$ \\
\hline País Vasco & 0,00381 & 0,00381 & $-0,01287$ & 0,00401 & $0,26125^{* *}$ \\
\hline Ceuta & $-0,02851^{* *}$ & $0,06716^{* *}$ & $-0,03793^{* *}$ & 0,03295 & $-0,03548$ \\
\hline Melilla & 0,00703 & $-0,03063^{*}$ & $-0,00540$ & $-0,03328^{* *}$ & $-0,44061^{* *}$ \\
\hline Constante & $0,09477^{* *}$ & 0,02831 & $0,10957^{* *}$ & 0,05017 & \\
\hline N. Obs. & 16.167 & 16.167 & 16.167 & 16.167 & \\
\hline Test F & 23.17 & 67.66 & 20.93 & 27.78 & \\
\hline $\mathrm{R}^{2}$ & 0,1181 & 0,2098 & 0,0980 & 0,0944 & \\
\hline
\end{tabular}

Nota: Estimación basada en los pesos muestrales de la ENS-2003. Errores estándar de los coeficientes del MLP robustos y los relativos a los índices de concentración se han calculado vía 'Bootstrapping». El número de replicas es de 100. Categorías omitidas: mujeres de 16-29 años, soltero, educación superior, empleado sector privado, fumador, sedentario, residente en Andalucía. Coeficientes estadísticamente significativos al 10\% ( ${ }^{*}$ ) y significativos al $5 \%(* *)$. 
podemos comprobar que el (log) de la renta neta equivalente ejerce mayoritariamente un esperado efecto negativo y estadísticamente significativo sobre la probabilidad de padecer cada dolencia crónica en este país (excepto para la hipertensión arterial). En términos de elasticidades, un aumento de la renta del $1 \%$ se traduce en una reducción en la probabilidad de padecer diabetes de 0,193 puntos porcentuales, en 0,149 para las enfermedades del corazón y una caída de 0,115 puntos para el colesterol elevado.

Por lo que respecta a las variables demográficas las estimaciones reflejan los datos de la Tabla 1 al evidenciar que la probabilidad de incurrir en cada problema crónico crece notablemente con la edad. Sin embargo, hay algunas diferencias por sexos. Respecto a la categoría de referencia, los resultados indican que en el segmento de mayores (población de 65 y más años) es más alta la probabilidad de contraer hipertensión arterial y tener el colesterol elevado en el colectivo de mujeres $(0,344$ y 0,184 , respectivamente) que entre los hombres $(0,215$ y 0,078$)$. En cambio, para las enfermedades del corazón y la diabetes apenas habría diferencias de género para este grupo de edad. Un patrón similar es el derivado de la situación de estar casado: esta condición conlleva una mayor -y estadísticamente significativa- probabilidad de padecer las cuatro enfermedades crónicas analizadas $(0,015$ en enfermedades del corazón, 0,036 en hipertensión, 0,018 en diabetes y 0,033 en colesterol elevado) en comparación con el soltero. Además nuestras estimaciones ponen de relieve que la situación de viudedad tiene incluso un mayor impacto en la probabilidad de sufrir del corazón o hipertensión arterial que el estar casado $(0,061$ y 0,05 , respectivamente) y la condición de divorcio (o separado legalmente) sobre la probabilidad de tener diagnosticado enfermedades del corazón o diabetes $(0,042$ y 0,030$)$.

Al igual que en la mayor parte de los estudios de este tipo, nuestros resultados apuntan (a excepción de las enfermedades del corazón) la existencia de un claro gradiente educativo en España en la incidencia de las restantes condiciones crónicas analizadas. Por consiguiente, los individuos entrevistados menos educados están relativamente más expuestos a una mayor probabilidad de declarar hipertensión arterial $(0,049)$, diabetes $(0,039)$ y colesterol elevado $(0,037)$ que los que poseen estudios superiores. Igualmente relevantes son los resultados que se refieren a la actividad económica de los individuos: el tener reconocida una pensión de invalidez aumenta las probabilidades de padecer un problema crónico o de larga duración (casi un 10\% para la hipertensión arterial y el colesterol elevado; $7 \%$ en enfermedades del corazón) al igual que tener la condición de pensionista - a excepción de la diabetes- aunque en menor grado en comparación con la categoría de referencia (empleado del sector privado). Por su parte, es interesante destacar que estar fuera del mercado laboral como inactivo (estudiantes, labores del hogar, voluntariado, etc.) o bien estar desempleado no tiene impacto alguno sobre la ocurrencia de declarar enfermedad crónica, como tampoco la tiene el hecho de estar bajo el estatus de servicio doméstico, empleado como autónomo o en una institución pública a excepción, en este último caso, de declarar tener dolencias crónicas del corazón. 
En cuanto a los estilos de vida, las estimaciones de la Tabla 3 muestran consistentemente que el sedentarismo eleva las probabilidades de declarar la afectación de enfermedades crónicas. A excepción del colesterol elevado, aquellos que declaran que ejercen una actividad física por mínima que sea (bien en el trabajo o en casa) tienen menores probabilidades de contraer dolencias crónicas en comparación al entrevistado que practica el sedentarismo. Al contrario, tanto el individuo que declara que no ha fumado nunca o lo ha hecho en el pasado derivan una mayor y estadísticamente significativa probabilidad de padecer enfermedades del corazón e hipertensión arterial, comparado al fumador habitual. De las estimaciones de la esta tabla no parece que los datos nos permitan derivar un patrón muy claro respecto las variables regionales.

\subsection{Estimación de los indices de desigualdad}

La misma Tabla 3 muestra también la estimación de los índices de concentración calculados para cada uno de los regresores $\left(\mathrm{C}_{\mathrm{k}}\right)$ respecto de la renta para las cuatro ecuaciones de las enfermedades crónicas. La inferencia estadística basada en técnicas 'bootstraping' nos advierte que todos esos índices son significativamente distintos de cero a excepción de los correspondientes a las variables divorciado, moderada y alta actividad física, residir en Aragón, Galicia y Ceuta. El índice de concentración (IC) del logaritmo de la renta equivalente $(0,0458)$ muestra una distribución desigual de la renta neta en favor de las decilas más altas de la escala de renta. En relación a los determinantes demográficos como la edad y el sexo, nuestras estimaciones evidencian que la población mayor (65 y más años) se concentra en los grupos de renta baja, aunque la desigualdad de la renta en ese segmento de población aparece más pronunciada en el colectivo de mujeres. Como cabía esperar, los españoles adultos con mayor nivel educativo están fuertemente concentrados en la parte alta de la distribución de la renta, mientras que lo contrario es cierto para las personas con poca educación (estudios primarios y/o sin estudios o analfabetos) ${ }^{15}$. Del mismo modo, en términos del estado civil los datos inequívocamente nos informan que la renta está desigualmente repartida en contra de los viudos, dado que buena parte de los ingresos de este colectivo está formado por rentas no contributivas. En la misma línea, los IC de la Tabla 3 muestran que los colectivos de pensionistas, inválidos, desempleados e inactivos al tener menores ingresos se concentran en la parte baja de la distribución de la renta, mientras que la población ocupada se concentra entre las rentas elevadas (cf. García-Gómez y López, 2004a,b y Costa-Font y Gil, 2008b). Finalmente, los resultados hallados validan la, por otro lado conocida, existencia de fuertes disparidades en la distribución de la renta a nivel regional en España.

Por último, la Tabla 4 presenta en las dos primeras filas: a) las estimaciones de los IC de la probabilidad de sufrir una dolencia crónica y b) los índices de desigual-

15 Estas características han sido, por otro lado, demostradas en el contexto de la EU (Van Doorslaer y Koolman, 2004). 
TABLA 4

ÍNDICES DE DESIGUALDAD Y CONTRIBUCIÓN DE LOS DETERMINANTES

\begin{tabular}{|l|c|c|c|c|}
\hline & $\begin{array}{c}\text { Enfermedades } \\
\text { Corazón }\end{array}$ & $\begin{array}{c}\text { Hipertensión } \\
\text { Arterial }\end{array}$ & Diabetes & $\begin{array}{c}\text { ColesteroI } \\
\text { Elevado }\end{array}$ \\
\hline IC & $-0,2237$ & $-0,1937$ & $-0,2389$ & $-0,1332$ \\
I $^{*}=$ IC-IC* & $-0,1160$ & $-0,0858$ & $-0,1145$ & $-0,0606$ \\
\hline Contribución: & $\%$ & $\%$ & $\%$ & $\%$ \\
Renta & $3,04 \%$ & $1,58 \%$ & $3,70 \%$ & $3,96 \%$ \\
Demografía & $48,15 \%$ & $55,72 \%$ & $51,96 \%$ & $54,50 \%$ \\
Estado civil & $11,83 \%$ & $3,81 \%$ & $3,60 \%$ & $-5,64 \%$ \\
Educación & $6,75 \%$ & $16,65 \%$ & $27,74 \%$ & $29,01 \%$ \\
Actividad económica & $28,13 \%$ & $17,39 \%$ & $7,13 \%$ & $19,62 \%$ \\
Estilos de vida & $2,91 \%$ & $0,45 \%$ & $2,76 \%$ & $-7,16 \%$ \\
Regional & $-0,80 \%$ & $4,41 \%$ & $3,11 \%$ & $5,72 \%$ \\
\hline
\end{tabular}

dad potencialmente evitable, I*, para cada una de ellas. Como cabía esperar, los IC de la probabilidad de padecer cada enfermedad crónica sobre la renta son negativos y estadísticamente significativos: $-0,224$ para las enfermedades del corazón; $-0,194$ para la hipertensión arterial; $-0,239$ para la diabetes y $-0,133$ para el colesterol elevado. Estos índices nos están, por tanto, indicando la existencia en la población adulta española de importantes desigualdades relacionadas con la renta o el SES en la probabilidad de sufrir cada enfermedad crónica estudiada, esto es, dichas enfermedades se concentran en los segmentos más pobres de la población ${ }^{16}$. En relación al grado de desigualdad relacionada con la renta del tipo "potencialmente evitable» $\left(I^{*}=\right.$ IC-IC*) o no explicada ni por la edad ni el sexo de la población, los resultados apuntan en la dirección que dicha desigualdad I* todavía sería substancial. En otras palabras, el peso del componente demográfico -a menudo considerado como inevitable- en el grado de desigualdad de la probabilidad de incurrir en estas enfermedades crónicas es francamente hegemónico, en concordancia con el hecho que dichas condiciones son mayoritariamente enfermedades propias del envejecimiento de la población.

\subsection{Descomposición de la desigualdad}

La Tabla 4 también nos informa sobre la descomposición de la contribución de cada variable explicativa al grado de desigualdad relacionado con la renta para cada condición crónica. De nuevo surgen algunos hallazgos interesantes. Como se ha comentado previamente, las variables demográficas, pero muy especialmen-

16 Resultados análogos son los hallados por Costa-Font y Gil (2008b) cuando analizan las desigualdades económicas en el estado de depresión de la población adulta. Véase nota al pie número 2. 
te las que se refieren al colectivo de personas de 65 y más años, son los determinantes más significativos que explicarían el grado de desigualdad observado en contra de los individuos situados en la parte baja de la escala de la renta (entre el 48 y el 56\%). Por tanto, gran parte de las desigualdades estimadas en la probabilidad de padecer enfermedades crónicas se debe al natural envejecimiento de la población. En cuanto al nivel de educación y la actividad económica como determinantes de la desigualdad, los resultados muestran incidencias dispares dependiendo de la enfermedad crónica considerada. Así, la educación (singularmente el bajo nivel educativo de los entrevistados) juega un papel incluso más relevante que el estatus económico tanto en el caso de la diabetes (28\%) como en el colesterol elevado (29\%). En cambio, para el caso de las enfermedades del corazón la condición económica de tener una pensión reconocida es más crucial para explicar la desigualdad observada que el propio nivel educativo (28\%); mientras que ambos determinantes son igual de efectivos en relación a la desigualdad en la probabilidad de declarar hipertensión arterial. Es igualmente notoria la contribución del estado civil, más bien la situación de viudedad, al grado de desigualdad estimada de las enfermedades del corazón (12\%). En consecuencia, vale la pena resaltar que la renta neta de los individuos ya no es un factor explicativo decisivo del grado de desigualdad medido, al contrario de lo que sucede cuando se analiza la desigualdad socioeconómica en estado general de salud o en salud mental (cf. García-Gómez y López 2004b y 2005 y Costa-Font y Gil, 2008b): la renta contribuiría menos de un $5 \%$ al grado de desigualdad estimada de la probabilidad de sufrir las enfermedades crónicas estudiadas. En otros términos, apenas llegaría a cambiar el patrón de desigualdades en la prevalencia de cada enfermedad crónica incluso si la renta se repartiese equitativamente entre la población, o si la elasticidad de cada enfermedad crónica respecto la renta fuese cero.

Finalmente, los restantes factores como los estilos de vida, el estado civil y las variables regionales se demuestra que ejercen un efecto residual como determinantes del IC de la probabilidad de incurrir en cada enfermedad crónica.

\section{Conclusiones}

Con el propósito de contribuir al debate y reflexión sobre los posibles factores explicativos de las desigualdades en salud, en este artículo hemos realizado una primera aproximación a la existencia de desigualdades socio-económicas en morbilidad. Hemos hallado evidencia empírica a favor de la existencia de un patrón de desigualdades socioeconómicas en morbilidad, concretamente en la probabilidad de padecer cuatro dolencias crónicas declaradas -enfermedades del corazón, hipertensión arterial, diabetes y colesterol elevado- responsables de una elevada tasa de mortalidad en la población adulta española, por medio de aplicar métodos recientemente elaborados (Van Doorslaer y Koolman, 2002) y el empleo de la Encuesta Nacional de Salud del 2003. De éstos resultados podemos concluir que la eviden- 
cia presentada al respecto respalda la hipótesis que existe un vector socioeconómico detrás de la exposición a los riesgos derivados de las enfermedades crónicas, lo que abre la puerta a una posible explicación de las desigualdades en el nivel de salud. Otra cuestión distinta es examinar a qué responde tal vector socio-económico y qué explica que existan diferencias significativas en los coeficientes de desigualdad, la expansión de los determinantes de la salud y el papel que cada determinante toma en cada caso. Así, los resultados alcanzados son consistentes con la idea de una asociación entre renta y morbilidad -o los riesgos «objetivos» para la saludde modo que la posición socio-económica puede estar detrás de una menor conducta preventiva por parte de la población, si bien esta cuestión debería ser objeto de un estudio más detallado.

Este trabajo, al descomponer el grado de desigualdad en sus potenciales determinantes, revela una incidencia mayoritaria de los factores demográficos - con una consiguiente menor capacidad de ser evitados por la política pública- siendo el nivel educativo y la actividad económica determinantes igualmente destacables, aunque heterogéneos según la condición específica analizada. En efecto, si bien para las enfermedades de corazón la actividad económica (esto es, la condición de pensionista) prácticamente explica cerca de un $30 \%$ de la desigualdad estimada (posiblemente asociada al estrés) siendo el efecto de la educación mucho menos relevante, en cambio para el colesterol y la diabetes el nivel educativo explica también cerca de un $30 \%$ de la desigualdades respectivamente, lo que es consistente con la existencia de desigualdades en el acceso a la información sanitaria y en su prevención. Por último, dado que el nivel de renta apenas influye en dichas desigualdades, una mera transferencia de recursos no necesariamente supondría una reducción de la desigualdad en salud.

Es importante reseñar que los resultados deben tomarse con cautela ya que existen algunos sesgos a tener en cuenta. Un problema potencialmente relevante de nuestro ejercicio tiene que ver con la naturaleza de los datos o probabilidad de revelación del encuestado de una dolencia. El hecho de declarar un problema de salud no sólo depende de la presencia de una dolencia crónica, sino que también de otros factores individuales como el grado de conocimiento del problema, la capacidad de recordarlo, las consecuencias de la enfermedad sobre la vida diaria, la frecuencia de visitas al doctor, etc., factores que pueden variar según la edad, el nivel de estudios o la renta. No cabe duda, que estas observaciones deben estar sesgando nuestras estimaciones y cuestionando de algún modo su validez. Sin embargo, a favor de nuestros hallazgos cabría hacer notar que existe evidencia que manifiesta que los datos declarados por los entrevistados (sobretodo en problemas del corazón y diabetes) serían bastante parecidos a los basados en exámenes médicos (Goldman et al., 2003), y para el caso de la hipertensión arterial el grado de acuerdo sería moderado pudiéndose emplear la información de encuestas con cierta cautela (Tormo et al., 2000). 


\section{Referencias bibliográficas}

[1] ABASOLO, I. (1998): «Equidad Horizontal en la Distribución del Gasto Público en Sanidad por Grupos Socioeconómicos en Canarias. Un Estudio Comparado con el Conjunto Español». Hacienda Pública Española, 147: 3-27.

[2] COSTA-FONT, J. (2005): «Inequalities in Self-Reported Health within Spanish Regional Health Services: Devolution Re-examined?» International Journal of Health Planning and Management; 20(1): 41-52.

[3] COSTA-FONT, J. y GIL, J. (2008a): «What Lies Behind Socio-Economic Inequalities in Obesity in Spain? A Decomposition Approach». Food Policy 33, 61-73.

[4] COSTA-FONT, J. y GIL, J. (2008b): «Would Socio-Economic Inequalities in Depression Fade Away with Income Transfers?». Journal of Happiness Studies, DOI 10.1007/s10902-008-9088-3.

[5] COSTA-FONT, J. y GIL, J. (2008c): «Exploring the Pathways of Inequality in Health, Access and Financing in Decentralised Spain», Documento de Trabajo de FEDEA 2008-13.

[6] DALSTRA, J.A.A., KUNST, BORRELL, C., BREEZE, E., CAMBOIS, E., COSTA, G., GEURTS, J.J.M., LAHELMA, E., VAN OYEN, H., RASMUSSEN, N.K., REGIDOR, E., SPADEA, T., and MACKENBACH, J.P. (2005): «Socioeconomic Differences in the Prevalence of Common Chronic Diseases: An Overview of Eight European Countries», International Journal of Epidemiology 34(2): 316-326.

[7] DEATON, M. (2002): «Policy Implications of the Gradient of Health and Wealth». Health Affairs 21(2).

[8] EVANS, R. (2002): «Interpreting and Addressing Inequalities in Health: From Black to Acheson to Blair to,...?». Office of Health Economics, London.

[9] GARCÍA-GÓMEZ, P. y LÓPEZ, A. (2004a): «The Evolution of Inequity in the Access to Health Care in Spain: 1987-2001». UPF Economics Working Paper 756.

[10] GARCÍA-GÓMEZ, P. y LÓPEZ, A. (2004b): «Regional Differences in Socio-Economic Health Inequalities in Spain». UPF Economics Working Paper 757.

[11] GARCÍA-GÓMEZ, P. y LÓPEZ, A. (2005): «Socioeconomic Inequalities in Health in Catalonia». Hacienda Pública Española/Revista de Economía Pública, 175(4/2005): 103-121.

[12] GOLDMAN, N., LIN, I.F., WEINSTEIN, M. y LIN, Y.h. (2003) «Evaluating the Quality of Self-Reports of Hypertension and Diabetes». Journal of Clinical Epidemiology 56(2): 148-154.

[13] JENKINS, (1988): «Calculating Income Distribution Indices from Micro-Data». National Tax Journal; 41(1): 139-142.

[14] KAKWANI, N.C., WAGSTAFF, A. y VAN DOORSLAER, E. (1997): «Socioeconomic Inequalities in Health: Measurement, Computation and Statistical Inference». Journal of Econometrics; 77(1): 87-104.

[15] KUNST, A.E., BOS, V., LAHELMA, E., BARTLEY, M., LISSAU, I., REGIDOR, E., MIELCK, A., CARDANO, M., DALSTRA, J.A.A., GEURTS, J.J.M., HELMERT, U., LENNARTSSON, C., RAMM, J., SPADEA, T., STRONEGGER, W. y MACKENBACH, J.P. (2005): «Trends in Socioeconomic Inequalities in Self-Assessed Health in 10 European Countries», International Journal of Epidemiology 34(2): 295-305. 
[16] LINK, B. G. y PHELAN, J.C. (1995): «Social Conditions as Fundamental Causes of Disease». Journal of Health and Social Behavior 35:80-94.

[17] MACKENBACH, J.P. (2005): «Health Inequalities: Europe in Profile», Informe independiente comisionado y publicado por la presidencia del Reino Unido de la UE. Disponible en: http://www.fco.gov.uk/Files/kfile/HI_EU_Profile,0.pdf.

[18] MATHERS, C.D., BERNARD, C., IBURG, K.M., INOUE, M., MA FAT, D., SHIBUYA, K., STEIN, C. y TOMIJIMA, N. (2003) «The Global Burden of Disease in 2002: Data, Sources, Methods and Results». GPE Discussion Paper No. 54, Geneva: World Health Organization. Disponible en http://www.who.int/evidence.

[19] REGIDOR, E., GUTIERREZ-FISAC, J.L. y RODRÍGUEZ, C. (1995) «Increased Socioeconomic Differences in Mortality in Eight Spanish Provinces», Social Science and Medicine, 41: 801-807.

[20] RODRÍGUEZ, M., CALONGE, S. y REÑÉ, J. (1993): «Spain», en: Van Doorslaer, Wagstaff y Rutten (eds.) Equity in the Finance and Delivery of Health Care: an International Perspective, Health Services Research Series, Commission of the European Communities, OUP 1993.

[21] STOYANOVA, A. (2004): «Equity and Utilisation of Primary, Specialist and Dental Health Care Services in Spain», Tesis Doctoral 2004, Universitat de Barcelona.

[22] SUHRCKE, M., NUGENT, R.A., STUCKLER, D. y ROCCO, L. (2006): «Chronic Disease: an Economic Perspective». Oxford Health Alliance. Disponible en http://www.oxha.org.

[23] TORMO, M.J., NAVARRO, C., CHIRLAQUE, M.D. y BARBER, X. (2000): «Validation of Self-Diagnosis of High Blood Pressure in a Sample of the Spanish EPIC Cohort: Overall Agreement and Predictive Values». Journal of Epidemiology and Community Health, 54(3): 221-226.

[24] URBANOS, R. (2000): «Desigualdades Sociales en Salud y Efectividad Potencial de las Políticas Públicas: un Estudio Aplicado con Datos Españoles». Hacienda Pública Española, 154: 217-237.

[25] URBANOS, R. (2001): «Explaining Inequality in the Use of Public Health Care Services: Evidence from Spain». Health Care Management Science, 4: 143-157.

[26] VAN DOORSLAER, E. y KOOLMAN, X. (2004): «Explaining the Differences in Income-Related Health Inequalities Across European Countries». Health Economics; 13: 609-628.

[27] VAN DOORSLAER, E., KOOLMAN, X. y MASSERIA, C. (2004): «Equity in the Delivery of Inpatient Care in the European Union: A Pooled Analysis», Equity Project III, Working Paper 13. Department of Health Policy and Management, Erasmus University, Rotterdam.

[28] WAGSTAFF, A. y VAN DOORSLAER, E (2000): «Equity in Health Care Finance and Delivery», en Culyer, A.J y Newhouse, J.P (ed.). Handbook of Health Economics, Cap. 34, Elsevier Science, B.V.

[29] WAGSTAFF, A., VAN DOORSLAER, E. y WATANABE, N. (2003): «On Decomposing the Causes of Health Sector Inequalities with an Application to Malnutrition Inequalities in Vietnam». Journal of Econometrics; 112: 207-223.

[30] WILKINSON, R. (1997): «Health Inequalities: Relative or Absolute Material Standards». British Medical Journal, 314(7080): 591-95. 
[31] OMS-Organización Mundial de la Salud (2005): «Prevención de las enfermedades crónicas: una inversión vital». World Health Organization. Disponible en: http://www.who.int/chp/chronic_disease_report/part1/es/index.html.

[32] YARNELL, J., YU, S., McCRUM, E., ARVEILER, D., HAAS, B., DALLONGEVILLE, J. et al. (2005): «Education, socioeconomic and lifestyle factors and risk of coronary heart disease: the PRIME study», International Journal of Epidemiology 34(2): 268-275. 